\title{
Why Do We Need an Architectural Approach to
}

\section{Interoperability?}

\author{
Bernd Blobel $^{1,2,3}$, Frank Oemig ${ }^{3,4}$ \\ ${ }^{1}$ Medical Faculty, University of Regensburg, Germany \\ 2 eHealth Competence Center Bavaria, Deggendorf Institute of Technology, Germany \\ ${ }^{3}$ HL7 Germany, Cologne, Germany \\ ${ }^{4}$ Deutsche Telekom Healthcare and Security Solutions GmbH, Mülheim, Germany
}

\begin{abstract}
Objectives: Health systems are on the move to increasing complexity, distribution, autonomy, number of domains or disciplines involved, thereby requesting evolution of interoperability to support required communication and cooperation among those systems for meeting intended business objectives.

Methods: Information cycle model with its phases and phase transitions as well as systems theory are used to describe structure and processes of healthcare business cases and the interoperability levels for enabling the communication and cooperation between the principals involved.

Results: When focusing on interoperability between health information systems acting as principals in an ICT business case, different levels of contribution to the common business case, i.e. phases to the completion of the information cycle, provided by the principals can be distinguished. While the first two levels, sharing data related to the business case, and sharing information derived from those data to define the required business process actions, deal with the communication challenge of interoperability, just the third level of providing the required action according to the business case concerns its operational part.
\end{abstract}

\section{Correspondence to:}

Prof. Dr. habil. Bernd Blobel, FACMI, FACHI, FHL7, FEFMI

Medical Faculty, University of Regensburg

c/o HL7 Deutschland e. V.

Address: An der Schanz 1, 50735 Köln, Germany

E-mail: bernd.blobel@klinik.uni-regensburg.de
Such service delivery requires appropriate system architecture for meeting the service functional cooperation challenge. When extending the consideration beyond ICT systems towards real world business systems, the architecture of non-ICT systems regarding their structure and behavior must be represented to be shared as required in the business case as well. This system extension requires domain knowledge based interoperability for covering the domain-specific concepts and relations including the constraints to be applied. When not just considering the domain-specific context, but also the context of the individual user, personalized business systems are managed.

Conclusions: Advanced healthcare systems require not just communication standards for enabling interoperability, but also multi-domain, ontology-driven interoperability standards based on a generic reference architecture, that is also shortly presented in this paper.

\section{Keywords}

Interoperability level; information cycle; systems; standards; architecture

\section{EJBI 2016; 12(1):en3-en9}

received: April 19, 2016

accepted: April 25, 2016

published: May 20, 2016

\section{Introduction}

Paradigm changes in health systems, discussed in several other papers in detail, require the advancement of the underlying interoperability paradigm [1, 2, 3]. The interoperability definition HL7 has originally referred to is the one provided at Merriam-Webster as "the ability of a system (as a weapons system) to use the parts or equipment of another system" 4. With advancing its communica- tion standards, HL7 has moved to the information and communication technology (ICT) related interoperability definition of IEEE: "Interoperability is the ability of two or more systems or components to exchange information and to use the information that has been exchanged" 5 . This IEEE definition focuses on interoperability within the domain of information and communication technologies (ICT), however. Well-known is the saying that the problem in ICT solutions is the user in front of the de- 
vice, and another statement tells us that the solution is excellent but doesn't fit the business domain's requirements and the user expectations. Interoperability is not just a matter of - even semantically correct - communication between ICT system, but also an issue of appropriate cooperation of all principals involved. Thereby, principals comprise persons, organizations, devices, applications, or components as defined by the Object Management Group (OMG) 6]. Therefore, the interoperability scope must be extended beyond ICT, covering all stakeholders and their ICT-independent domains contributing to the business case in question. The newest HL7 SAIF interoperability definition "interoperability is the ability of two parties, either human or machine, to exchange data or information where this deterministic exchange preserves shared meaning" goes beyond the ICT domain by involving humans, but it is still restricted to the communication paradigm [7]. The paper introduces different interoperability levels and the role of architectures for advancing interoperability beyond the aforementioned limitations.

\section{Methods}

\subsection{Information Cycle and Related Interoperability Level}

For practicing communication and cooperation, sharing of data as well as information is necessary, irrespective of whether this information sharing concerns ICT processes, existence of shared knowledge or verbal and nonverbal communication between living entities. Therefore, the information cycle model [8] is deployed in our approach (Figure 1p).

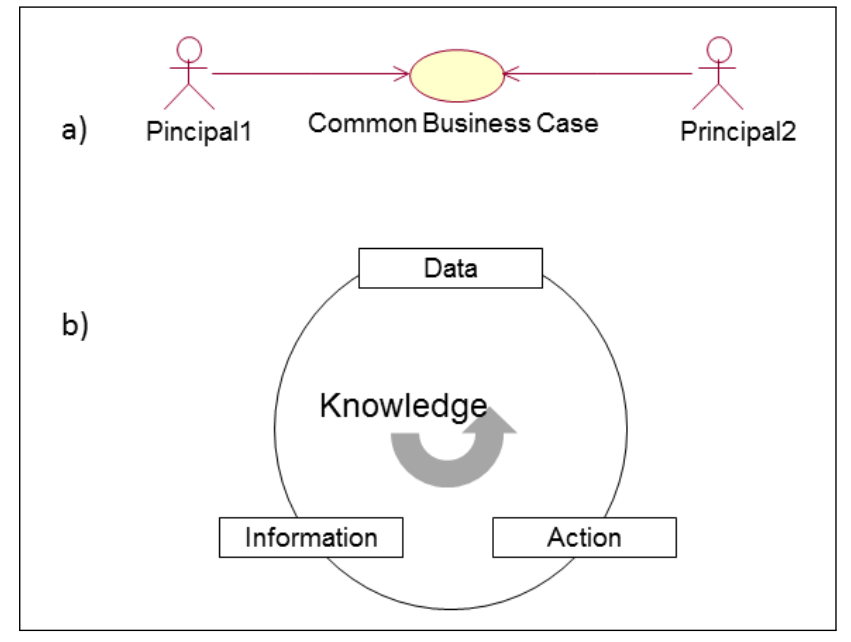

Figure 1: Information cycle (after [8], changed).

The business case serves the creation, transformation, preservation of business system components according to the business objectives. For realizing a common business case by two communicating and cooperating principals involved as described in Figure 11 , all objects and related processes including the environmental as well as con- textual conditions resulting in data must be considered. Those data have to be interpreted resulting in semantically correct information. That information must guide the principals in taking appropriate action. In healthcare, those phases are called observation, diagnosis, and therapy. The phases realized in the information cycle deploy existing or emerging knowledge, skills and capabilities, which have to be shared among the principals either a priori or as part of the communication and cooperation process. In the context of health informatics interoperability challenges, ICT mediated communication and cooperation is usually considered.

\subsection{Systems Approach to Interoperability}

A system is an ordered composition of interrelated elements, separated from the environment it interacts with. A system's architecture describes the systems elements (components), their functions and interrelations. So, it represents the structural, functional and behavioral aspects of a system. Rules for selecting components and functions as well as constraints of the relations according to a business case are called policies. Policies define the intended behavior of a system.

A business case is a system, which provides an intended outcome according to the business objective based on given or appropriately selected inputs and eventually also specific controls, or feedbacks.

\section{Results}

When focusing on the information cycle, the following interoperability levels between ICT systems are realized (Table 1).

Technical interoperability deals with the connectivity of systems. Structural interoperability and syntactic interoperability address different levels of data exchange by defining either simple data units or more complex rules for structuring the data stream. Semantic interoperability concerns advanced information sharing based on common information models and common terminologies/ontologies to consistently represent the concepts relevant in the business case. Current communication standards cover this continuum by addressing first the connectivity challenge (ISO/OSI lower layers protocols), followed by the data interchange challenge (EDI, HL7 v2), and thereafter by the information exchange challenge (HL7 v3). All the aforementioned interoperability levels cannot guarantee practical interoperability in the context of the business case, as they support the communication of information as first part of the IEEE definition, but not its use. They just support principals to act properly. Communication protocols are unable to perform operations and to take any action. 
Table 1: Interoperability levels between ICT systems [1.

\begin{tabular}{ll}
\hline Interoperability Level & Instances \\
\hline Technical interoperability (0) & Technical plug\&play, signal - \& protocol compatibility \\
Structural interoperability (1) & Simple EDI, envelopes \\
Syntactic interoperability (1) & Messages, clinical documents, agreed vocabulary \\
Semantic interoperability (2) & Advanced information sharing, common information \\
Organizations/Service interoperability (3) & models and terminology/ontology \\
\hline
\end{tabular}

\subsection{The ICT Systems Architecture Approach}

The first step to overcome those limitations is the deployment of common information in an informational representation of the business process, i.e., the specification of active objects, active resources, or service-oriented architecture solutions. Such service interoperability requires common or interrelated business processes, ruling the aggregation of activities to actions and complex processes, but also architectural principles for ICT systems design and implementation. Related standards meet the service functional cooperation challenge. Examples for such solutions are the OMG's Common Object Request Broker Architecture (CORBA), HL7 FHIR resources, or The Open Group's Service Oriented Architecture with their services (CORBA services, web services, etc.). Requirements for designing such systems are formulated in functional or non-functional requirements specified, e.g., the ISO/HL7 10781 HL7 Electronic Health Records-System Functional Model and ISO/HL7 16527 PHR System Functional Model. For representing them, ICT ontologies are deployed.

For ensuring comprehensive interoperability, the functional and behavioral aspects of the real world business system must be managed in coincidence with the business objectives, and the business processes to be performed for achieving them. In consequence, the technical interoperability definition must advance to "interoperability describes ability and capability to cooperate for achieving common goals or business objectives" [1, 9. Related standards deal with the knowledge-based interoperability challenge of interrelated business domains, represented using the domain-specific terminologies and underlying ontologies. In cases of human principals' involvement, social and psychological factors such as motivation and willingness must be considered as well. Related standards meet the skills based interoperability challenge. Summarizing the aforementioned statements, only the standards types mentioned in this section should be called interoperability standards.

\subsection{Architecture Models and Frameworks}

When talking about architectures, we will be confronted with a bunch of different approaches. Even within the ICT domain, many different architecture models and frameworks addressing different aspects of the ICT system have to be considered, as shown in the OPEN Process Framework Architecture (Figure 2] 10].

The process is getting even more challenging when extending the consideration to business cases (BCs) with essential non-ICT process parts. The resulting interoperability scenario is presented in Figure 3.

In the gray-shaded ICT system part, connectivity (0), interface (IF) mediated data exchange (1), sharing of semantics at data representation (DR) level (2), and services sharing at application (APP) level (3) are realized. Beyond ICT, domain-domain interoperability is managed based on sharing domain knowledge to cover domainspecific concepts and relations including constraints (4), thereby harmonizing the ontologies of the domains or disciplines involved. When not just considering the domainspecific context, but also the context of the individual user, personalized systems are managed (5).

For managing business systems according to a business case as described in Figure 3 , the system must be properly represented regarding structure and behavior, also reusing and correctly representing the domains knowledge using the domain ontologies.

In [3], the authors have introduced and comparatively evaluated the following architectural models and frameworks (references to the listed architectural models and frameworks can be found in [3]):

- Zachman Framework for Enterprise Architecture;

- OMG's Model Driven Architecture;

- The Open Group Reference Architecture for SOA;

- OASIS Reference Architecture for SOA;

- ISO/IEC/IEEE 42010 Systems and software engineering - Architecture description;

- US Federal Enterprise Architecture Framework including the FEAF Consolidated Reference Model (CRM);

- ISO 10746 Information technology - Open Distributed Processing - Reference Model;

- The Open Group Architecture Framework (TOGAF) including the TOGAF 9 Architecture Development Method (ADM); 
- ISO/EN 19439 Enterprise integration - Framework for enterprise modelling;

- Web Services Architecture;

- HL7 Clinical Document Architecture;

- HL7 FHIR Resources, and others.

One of the very few models going beyond a strict restriction on ICT is the Zachman Framework for Information Systems Architecture, later on generalized towards the Zachman Framework for Enterprise Architecture [12, 13. It is a two-dimensional classification schema for descriptive representations of an enterprise, thereby using a mixture of presentation means from natural language representations through Entity-Relationship (ER) Diagrams and Chen Diagrams up to Bachman Diagrams. Also symbolic logic deploying either the predicate calculus or the conceptual graph notation has been discussed
[14. The Zachman Framework for Enterprise Architecture defines neither a development process or a development metho-dology nor specific deliverables. It doesn't help identifying and managing dependencies [15.

\subsection{A Domain-Crossing Interoperability Reference Architecture}

There are different types of standards and specifications harmonizing approaches to domains and their concerns: Norms, standards, or publicly available specifications (PAS). Regarding the legal force, we can distinguish de jure, de facto, ad hoc, consensus, and governmental standards. Some of the Standards Developing Organizations (SDOs) are specialized to a specific domain, while others address cross-domain or multi-disciplinary concerns. The latter are frequently governmentally accredited (e.g. CEN, NIST, DIN, NEN, or AFNOR) and

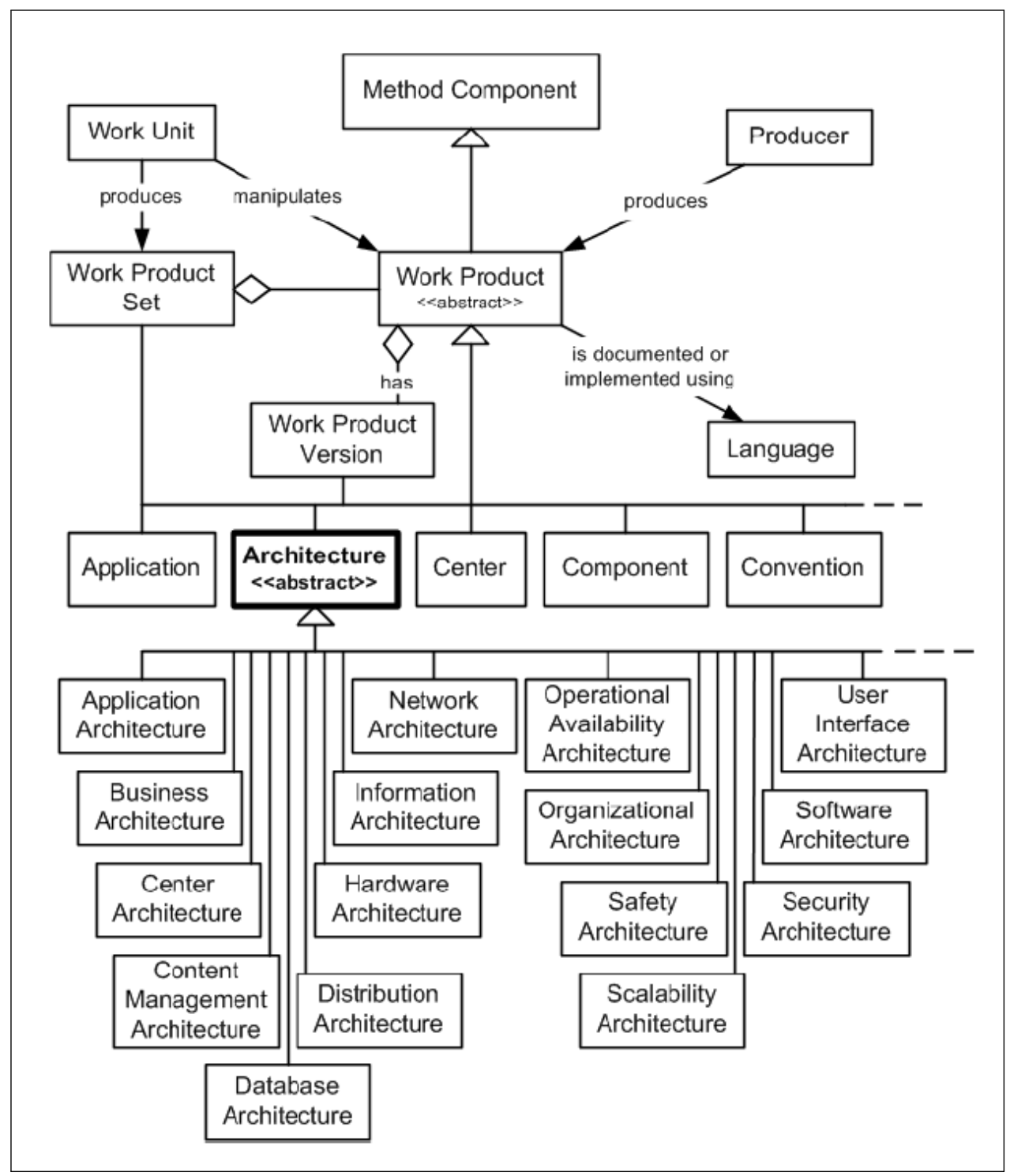

Figure 2: OPEN Process Framework Architecture [10]. 
domain-specifically structured in domain-specific committees and related working groups.

For modeling real world business systems, the following good modeling practice principles have been introduced [16: a) The domains of discourse, the real world business objectives, and the domain experts must be defined, where the latter have to be dominantly involved. b)

Within this involvement, those stakeholders define the provided view of the model as well as the way of structuring and naming the concepts of the problem space.

Therefore, standards for agriculture are developed by farmers or agro-engineers, specifications for pharmacological formulas are created by pharmacists, standards in machine construction are developed by mechanical engineers, etc. Information technology specifications are usually and correctly elaborated by IT experts. However, standards for managing and optimizing business processes in health and social care including rules for practicing medicine are mistakenly mainly defined by informaticians. It is not just the lack of domain-specific knowledge and experiences what is frequently missing in SDOs acting in the health and social care informatics but also bioinformatics. Also attempts in enforcing the informatics domain specific methodologies and presentation styles cause frequently big trouble. It is impossible to represent the highly complex, highly dynamic, multidisciplinary/ multi-domain healthcare system by one domain's terminology or even by using ICT ontologies (such as archetypes, HL7 RIM, Zackman
Framework, etc.) and enforcing that those styles are applied by the other domains as well.

As application-agnostic communication standards have been successfully developed to enable crossapplication data and information exchange, domainindependent reference systems have to be developed to bridge between different domains and their real world business systems.

There is a long tradition in homogenously representing things across different domains practiced in philosophy, using abstract representation means of mathematics and especially logics. Another younger tradition with the same objective evolved in system theory, later on combined with cybernetics. Based on those streams, an application domain and technology agnostic approach has been developed in the early nineties at the German CORBA group the first author has been involved in. This approach of a generic composition/decomposition layer model has been further matured by the first author towards the Generic Component Model (GCM) [1, 17. The outcome is not a layer model anymore, but a three-dimensional representation of a system, addressing the system components composition/decomposition, the representation of different domain-specific perspectives on the system represented by domain experts using domain-specific terminologies and their underlying ontologies. With that model, all use case specific different domains contributing to a real world system to realize a specific business case can be represented and interrelated. Thereby, the different domain-specific representations must be linked to

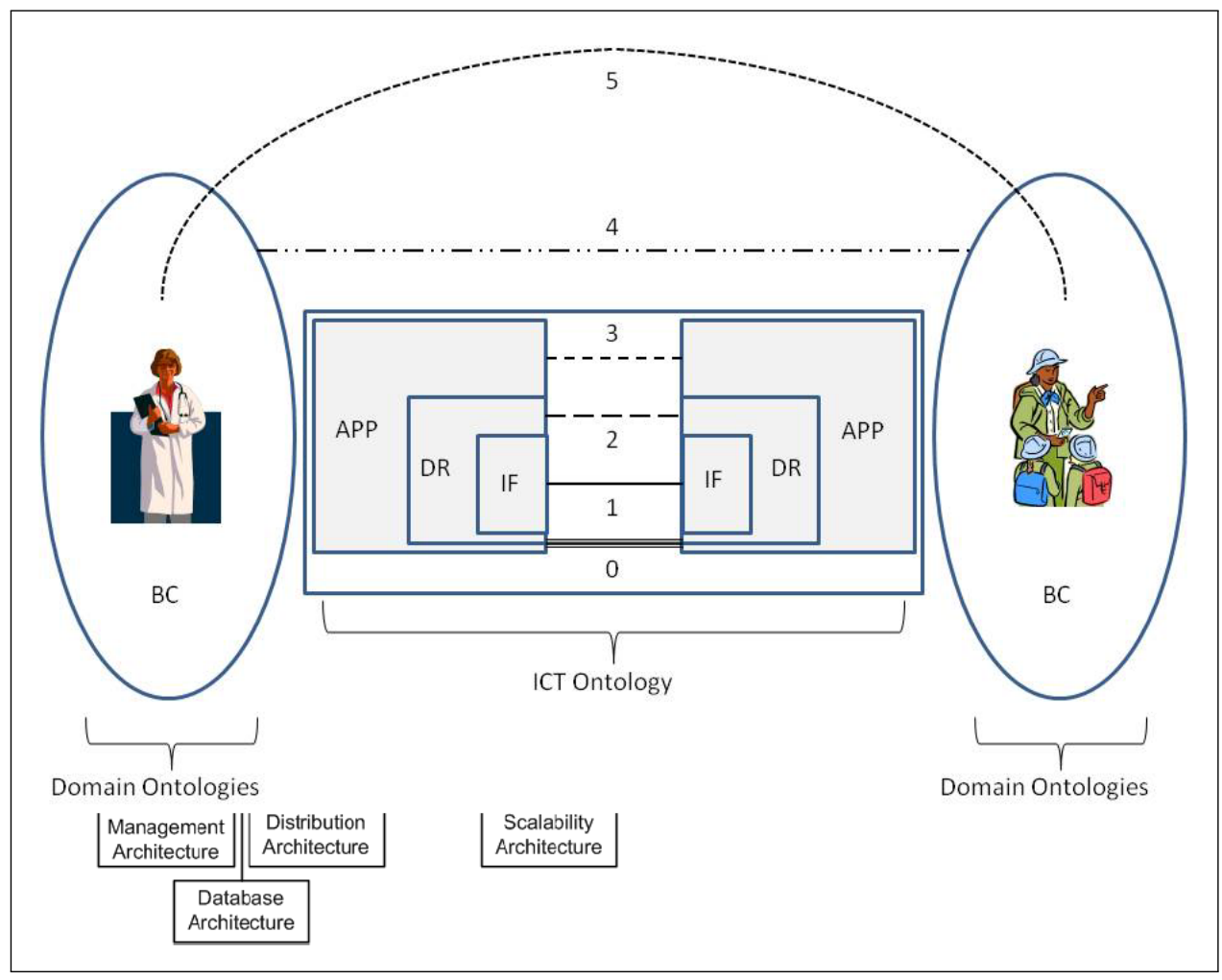

Figure 3: Comprehensive interoperability scenario [11. 
the same real world component presented in the abstract reference architecture model. This process is called reengineering the domains in a reference architecture model. The formally represented business system can be easily transformed into an ICT system supporting the real world scenario by deploying the Rational Unified Process or the related standardized representation through the ISO/IEC 10746 RM ODP [18. This development process dimension provides the third axis to build the GCM cube.

In summary, the representation of all the domains in components of an abstract uniform component model architecture allows a formalized and harmonized reengineering of those domains into an Interoperability Reference Architecture Model as shown in Figure 4 to describe their interoperability.

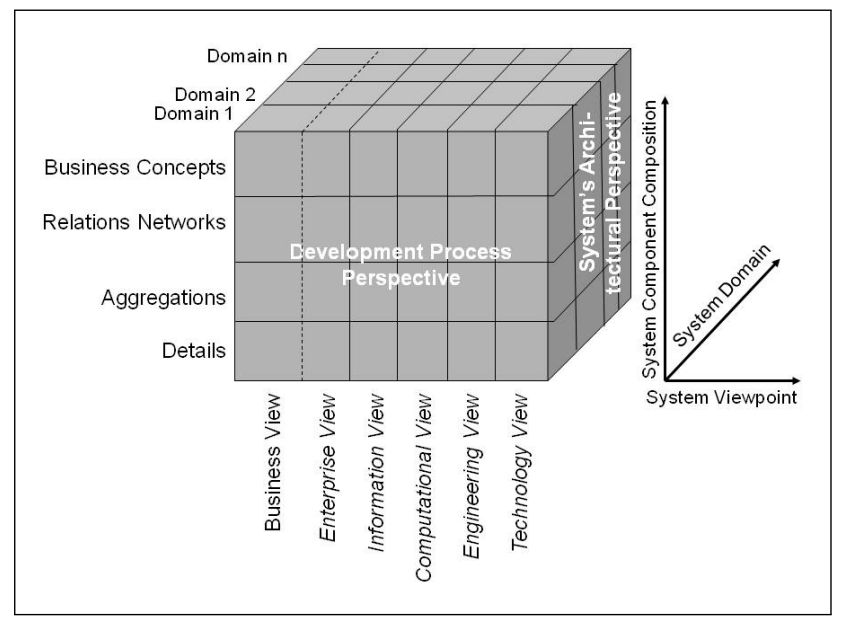

Figure 4: Interoperability Reference Architecture Model.

Not just related to the stakeholder groups involved in the system modeling process, but also related to the modeling process itself, the good modeling practice principles must be followed. Those principles require capturing key concepts and key relations at a high level of abstraction first. Different abstraction levels should be used iteratively, where the first iteration is performed in a topdown manner to guarantee the conceptual integrity of the model. Thereby, design principles such as orthogonality, generality, parsimony, and propriety must be met [16].

\section{Discussion}

Currently, interoperability solutions are ICT focused. Most of them are restricted to the communication paradigm, thereby supporting just one or two phases of the information cycle model.

For guaranteeing that the intended actions in a business case for meeting the business objectives are performed by all principals engaged in the business system, the operational aspect of interoperability has to be managed appropriately. Hereby, the business systems behavior is relevant, described by the business system architecture and its response to environmental and contextual conditions. Therefore, only an architectural approach can enable comprehensive interoperability. However, the consideration must go beyond the ICT domain.

The representation of health and social care systems is especially challenging due to the complexity and interdisciplinary of those systems. Multi-disciplinary systems are characterized by the huge number of different domains involved and represented by domain experts using domain-specific methodologies, terminologies, and ontologies for correctly representing domain knowledge and deriving new domain-specific insights. Those domains must be correctly interrelated, i.e., according to the real world system architecture representing all the perspectives of the domains involved, to realize comprehensive interoperability. For this purpose, the right component at the right granularity level must be interrelated according to the concepts of the different domains perspectives on that real world system component.

The very few current approaches claiming to solve that problem primarily do this either on the basis of implicit knowledge or by using one domain's specific ontologies (e.g. ICT ontologies) and/or presentation tools (e.g. UML notation) experts from other, primarily addressed domains (e.g. medicine) or involved domains (e.g. jurisprudence) cannot understand. Overcoming the problems of that approach would require universal education in all domains, training in formalizing knowledge, as well as deployment of abstraction and representation style of the minority.

The solution out of the described dilemma is an abstract, systems theory based, ontology-driven Interoperability Reference Architecture Model, preserving ontologies, methodologies and ways of thinking of all the domains involved and automating the harmonization process. It is described in very detail in [19.

\section{Conclusions}

Comprehensive interoperability of complex, flexible, scalable, business-controlled, adaptive, knowledge-based, multi-domain intelligent systems must follow a systemsoriented, architecture-centric, ontology-based and policydriven approach. Interoperability is not just provided through specifications, but must be enforced at implementation level as well. This requires implementable specifications, tooling and platforms. FHIR, REST and Web Services are pushing this approach. However, one should never forget that ICT is not the matter of health and social care but a technology to support them.

\section{References}

[1] Blobel B. Architectural Approach to eHealth for Enabling Paradigm Changes in Health. Methods Inf Med. 2010; 49(2): 123-134.

[2] Blobel B. Paradigm Changes of Health Systems Towards Ubiquitous, Personalized Health Lead to Paradigm Changes of the Security and Privacy Ecosystems. International Journal on 
Biomedicine and Healthcare 2015;3,1:6-11. Blobel B. Architectural Approach to eHealth for Enabling Paradigm Changes in Health. Methods Inf Med. 2010; 49(2): 123-134.

[3] Blobel B, Oemig F. The Importance of Architectures for Interoperability. Stud Health Technol Inform. 2015;211:18-56. Blobel B. Architectural Approach to eHealth for Enabling Paradigm Changes in Health. Methods Inf Med. 2010; 49(2): $123-134$.

[4] Merriam-Webster. Dictionary. http://www.merriam-webster. com/dictionary/interoperability Blobel B. Architectural Approach to eHealth for Enabling Paradigm Changes in Health. Methods Inf Med. 2010; 49(2): 123-134.

[5] IEEE (1990) IEEE Standard Computer Dictionary: A Compilation of IEEE Standard Computer Glossaries, IEEE

[6] Object Management Group. Security Service Specification V 1.7. Needham: OMG; 2001.

[7] Health Level 7 International, Inc. SAIF Interoperability definition.

[8] Van Bemmel J, Musen M (Edrs.) Handbook of Medical Informatics. Heidelberg: Springer; 1997.

[9] Lopez DM, Blobel B. Enhanced Semantic Interoperability by Profiling Health Informatics Standards. Methods Inf Med. 2009;48,2:170-177.

[10] OPEN Process Framework Repository Organization (OPFRO). OPEN Process Framework Architecture. http://www.opfro.org/index.html?Components/ WorkProducts/ArchitectureSet/Architectures/ Architectures.html Contents

[11] Oemig F and Blobel B. Natural Language Processing Supporting Interoperability in Healthcare. In: Biemann C, Mehler
A (Edrs.) Text Mining - From Ontology Learning to Automated Text Processing Applications, 137-156. Series Theory and Applications of Natural Language Processing. Heidelberg: Springer-Verlag GmbH; 2014.

[12] Zachman JA. A framework for information systemsarchcitecture. IBM Systems Journal 1987; 26,3:276-292.

[13] Zachman JA. The Zachman Framework: Primer for Enterprise Engineering and Manufacturing. Zachman International 2003. http://www.zachmaninternational.com

[14] Sowa JF and Zachman JA. Extending and formalizing the framework for information systems architecture. IBM Systems Journal 1992; 31,3:590-616.

[15] Rosen M. MDA and the Zachman Framework. M2VP Inc. 2003.

[16] Lankhorst M, et al. Enterprise Architecture at Work - Modelling, Communication and Analysis. The Enterprise Engineering Series. Berlin Heidelberg: Springer-Verlag; 2009.

[17] Blobel B. Analysis, Design and Implementation of Secure and Interoperable Distributed Health Information Systems. IOS Press: Amsterdam, Berlin, Oxford, Tokyo, Washington DC; 2002.

[18] International Organization for Standardization. ISO/IEC 10746-2:1996 Information Technology - Open Distributed Processing - Reference Model: Foundations. Geneva: ISO; 1996. Revised to ISO/IEC 10746-2:2009 Information technology Open distributed processing - Reference model: Foundations.

[19] Blobel B. A System-Oriented, Architecture-Centric, Generic Reference Model to Enable Model and Standards Interoperability. Submitted to Methods of Information in Medicine. 